\title{
Automatic Segmentation of Mitral Leaflet Movement in Doppler Tissue M-Mode Ultrasound
}

\author{
Kasper Soerensen ${ }^{1}$, Samuel Emil Schmidt ${ }^{1}$, Peter Lyngoe Soerensen ${ }^{1}$, Anne-Sofie Korsager ${ }^{1}$, \\ Jacob Melgaard $^{1}$, Peter Soegaard ${ }^{2}$, Johannes Jan Struijk ${ }^{1}$ \\ ${ }^{1}$ Department of Health Science and Technology, Aalborg University, Denmark \\ ${ }^{2}$ Aalborg University Hospital, Denmark
}

\begin{abstract}
Introduction: Doppler Tissue M-Mode ultrasound facilitates a method for measuring both mitral and aortic valve opening and closing. These measures can be used when assessing global information about the cardiac cycle. The method depends on an operator to manually setting points in the ultrasound image to obtain the timing intervals. We developed an automatic segmentation algorithm that segmented the mitral leaflet movement line, to guide the clinician in this work.

Methods: The algorithm used a series of steps to reduce noise in the image, leaving only the region of interest left with the mitral leaflet movement line. Next the gradient of the image was calculated with a the Sobel method, creating a high gradient on the edge of the mitral leaflet movement line. Using a multidimensional weight matrix the mitral leaflet movement was tracked in the image. The automatic algorithm is validated against three human operators.

Results: In 19 of 29 images the correlation coefficients are larger than 0.8 between the algorithm and the mean of the operators. The median operator standard deviation within the 95\% CI was 5 pixels. The median rms error for the algorithm was 4 pixels.

Conclusion: The novel method of automated segmentation the mitral leaflet movement line has a high accuracy but depends on the quality of the image.
\end{abstract}

\section{Introduction}

Using Doppler Tissue M-Mode ultrasound imaging have previously been proven valuable when measuring cardiac timing intervals [1, 2]. Using an anatomic color Doppler Tissue M-Mode drawn across the mitral leaflet both Kjaergaard et al. [1] and Voigt et al. [2] were able to estimate aortic opening (AO) and aortic closure (AC) together with mitral opening (MO) and mitral closure (MC) see Fig 1. Timing of AO and AC is based on the color shift from red to blue in the line characterizing the mitral leaflet movement [2]. This shift in color is caused by the backwards movement of the mitral valve that follows AC [2].

Using the mitral valve leaflet for cardiac timings the isovolumic periods and left ventricular ejection time can be calculated [1]. These timings can be used to assess global information about the cardiac cycle, which is of value in e.g. patients with heart failure, aortic stenosis and essential hypertension with left ventricular hypertrophy [1].

Selecting the timing points for $\mathrm{AO}, \mathrm{AC}, \mathrm{MO}$ and $\mathrm{MC}$ is currently done manually by a clinician. This process is considered time consuming and will cause operator variability.

Therefore we developed a method for automatic segmentation of the mitral leaflet movement line in Doppler Tissue M-Mode ultrasound images. This novel method could be a guide for the clinician to help setting the timing points, thereby saving time and potentially reduce inter-operator variability.

\section{Method}

For the development of the algorithm a sample of 5 ultrasound images was used from the dataset consisting of a total of 29 images from 29 healthy subjects. These 29 images were selected from a dataset of 44 images, where 15 were considered useless due to poor quality. The images were all exported from the ultrasound database in DICOM format. The image size before cropping was 434 pixels in height and 636 pixels in length.

All image processing was done in Matlab 2015b with functionality from the "Image Processing Toolbox". In the validation of the algorithm all 29 images were used including the images used for algorithm development.

\subsection{Image quality}

The image quality varies among the different ultrasound images with respect to how clearly the mitral leaflet 
movement line is presented. Fig 1 and Fig 2 shows examples of two images one high quality image with a very clear line of movement and one of a poor quality respectively.

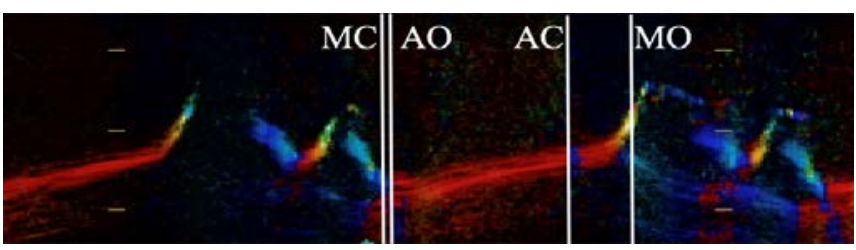

Fig 1 - Doppler Tissue Ultrasound Image with clear mitral leaflet movement line. Lines indicating $M O, M C, A O$ and $A C$ according to $[1,2]$

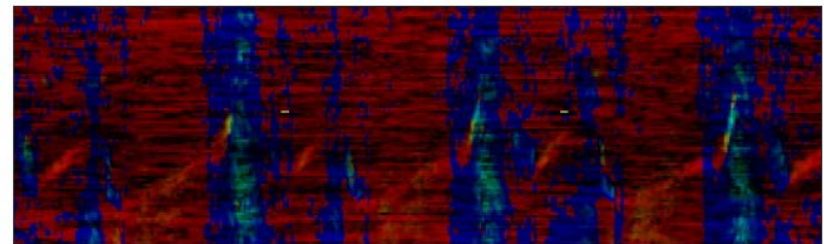

Fig 2 - Doppler Tissue Ultrasound Image where it is difficult to visualize mitral leaflet movement

In Fig 1 the movement of the mitral valve leaflet is easily recognizable when comparing to Fig 2. To the trained human eye, Fig 2 will still provide a line of movement that is visible, but the line is surrounded in color of the same intensity.

For development of the method images with both high and low quality were used in order to insure a general approach yielding an acceptable result for all images.

The method consists of a preprocessing part and a line tracking part.

\subsection{Preprocessing}

The preprocessing of the ultrasound images was done in order to remove as much background noise as possible and to define a region of interest (ROI) showing only the mitral valve movement line.

When exported from the ultrasound database the ultrasound image itself contains a lot of information unnecessary in this specific project. Because of this, the image is first manually cropped in an area around the leaflet movement, leaving an image size of approx. 100 pixels high and 520 pixels wide.

Next the blue and red color channels of the image were filtered with a 3-by-3 neighborhood median filter separately [3]. The intensities from the blue channel were added to the red, leaving the blue and green color channel empty, in order to obtain the highest intensity difference between background noise and the ROI, see Fig 3.

Subsequently the contrast was adjusted to remove background noise, see Fig 4. The image was then converted to a grayscale image and the gradient of the image was obtained with a Sobel gradient operator [3].

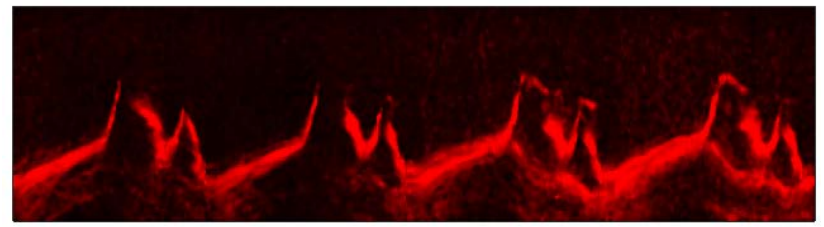

Fig 3 - Image from Fig 1 (different scaling) after filtering and appending the blue color channel to the red channel

As seen in Fig 4 the contrast between the background and the mitral valve movement is high.

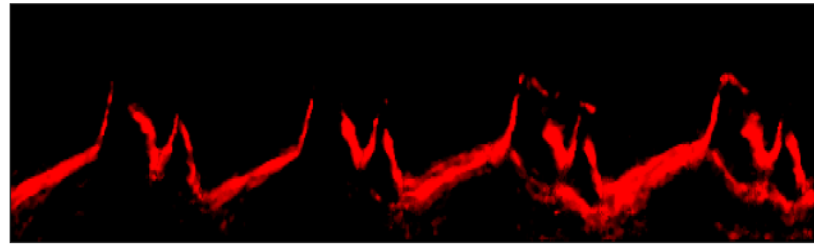

Fig 4 - Image from Fig 3 after adjusting for contrast

This yields a high positive gradient in the transmission between background and mitral valve movement, see Fig 5. The result of the gradient transformation is used in the final step line tracking algorithm.

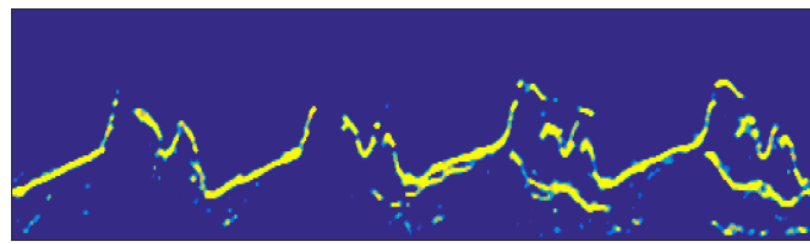

Fig 5 - Gradient image of Fig 4. High gradients are shown in yellow whereas low gradients are shown in blue

\subsection{Line tracking}

Following the preprocessing, an algorithm develop for tracking the mitral valve movement was applied. The algorithm uses a weighting matrix to weigh the intensities in the image and selecting the pixel with the highest intensity after this weighting. The $3 \mathrm{D}$ weight matrix is composed by three first order exponential functions. The objective was to weigh intensities located high in the image with a larger weight, than intensities located lower in the image. The weight matrix was created based on the images selected for algorithm development, which all showed the same pattern with respect to what pixel to choose next. As default the weight matrix is 5 pixels in the length-direction (x-direction) of the image and covers the whole height ( $\mathrm{y}$ direction) of the image. Fig 6 shows a visualization of the weight matrix.

The line tracking algorithm started by determining the pixel with the highest intensity in the first (most leftwards) column of the image. 


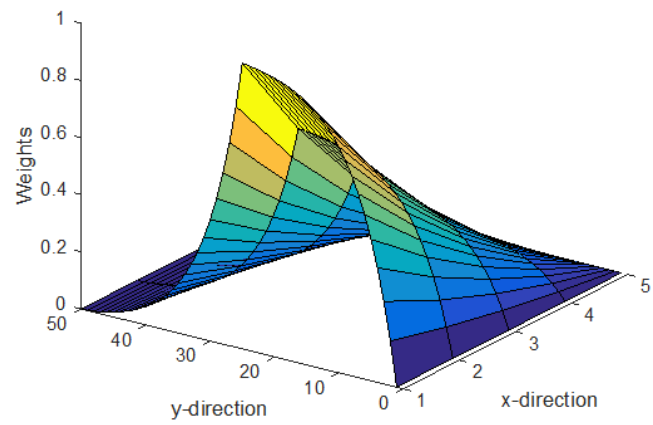

Fig 6 - Weight Matrix used in the line tracking algorithm. The ydirection in the image is the height of the image and the $x$ direction is the length. Highest weight located at $y=25$. Note that $y=0$ corresponds to the top of the image and $y=50$ to the bottom of the image.

The weight matrix was applied to the image from the next column onwards, positioned such that the highest weight was applied to the pixel in the same row, as the one just found. The pixel with highest intensity within the range of the weight matrix was then found. The weight matrix was then moved horizontally to the column with the pixel just found and moved vertically to align the highest weight, to the row of the found pixel. This was done repeatedly throughout the image.

The algorithm had a build-in fail detection ensuring that in the case of all the intensities in the columns being processed being zero, the length of the weight matrix was extended from 5 to 30 pixels to locate the next pixel.

At each step the location of the pixel with the highest intensity was recorded. These locations made up the final line describing the mitral valve movement. As the algorithm allowed locating pixels that were further away from each other than one column a linear interpolation was applied to create locations points for every column in the image. An example of the final line tracking is shown in Fig 7.

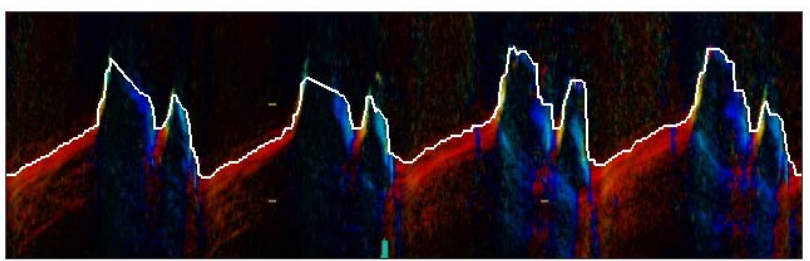

Fig 7 - Final result of the automatic segmentation shown as the white line

\subsection{Validation of the algorithm}

To validate that the algorithm had found the correct mitral leaflet movement line, three operators manually segmented parts of the movement line in the 29 images.
The segmentation was first done by one operator, selecting part of the mitral leaflet movement line and marked points in that part of the line. The line was marked, starting from just before MO and ending just after MC. This part is of interest as it is used in the methods in [1, 2]. The two other operators then marked the movement line, in the same time instances (x-coordinates) of the image, giving a total of three points for every time instance in every image. From these three points a mean point was calculated, describing a mean operator point. This was done for all points in all images.

For each image a root mean squared error (RMSE) was calculated, between the mean operator points and the points derived from the algorithm at the same time instances and between the individual operator and the mean operator points. Also the correlation coefficients between the algorithm and the operator mean points were calculated for all images, thus describing the agreement between operator and algorithm in relation to the waveform of the points.

\section{Results}

The results of the algorithm validation are shown in Tab 1. For 19 of the 29 images the correlation coefficient is above 0.8 indicating an good agreement of the waveform of the mitral leaflet movement line between mean operator segmentation and the algorithm. For 16 of the 29 images, the RMSE for the algorithm lies within two standard deviations of the operators mean.

Average operator standard deviation for all images was 4 pixels. Average and median operator standard deviation within the 95\% CI was 8 and 5 pixels respectively for all images. Average and median RMSE for the algorithm was 28 and 4 pixels respectively for all images.

\section{Discussion}

This paper describes a novel method for automatic segmentation and tracking of the movement line of the mitral valve. Visual inspection of the results of the algorithm visually shows a large variation in the quality of tracking of the movement line. The images acquired for this paper were very varying in quality, with some images clearly showing the movement line whereas the line was almost impossible to see in others. As assumed the quality of the line tracking is highly dependent on the image and how clearly and well defined the mitral valve movement line is. Especially how much noise is present around the mitral leaflet movement line is critical, as noise with the same intensity and color is difficult to remove, without removing the line that is of interest, with the method presented. 


\begin{tabular}{|c|c|c|c|}
\hline $\begin{array}{l}\text { Image } \\
\text { No }\end{array}$ & $\begin{array}{l}\text { Correlation } \\
\text { Coefficient }\end{array}$ & $\begin{array}{l}\text { Operator } \\
\text { RMSE } \\
\text { [pixels] }\end{array}$ & $\begin{array}{l}\text { Algorithm } \\
\text { RMSE } \\
\text { [pixels] }\end{array}$ \\
\hline 1 & -0.2914 & 5 & 36 \\
\hline 2 & 0.9988 & 4 & 1 \\
\hline 3 & 0.5263 & 12 & 45 \\
\hline 4 & 0.9933 & 4 & 5 \\
\hline 5 & 0.998 & 2 & 1 \\
\hline 6 & $\mathrm{n} / \mathrm{a}$ & 2 & 47 \\
\hline 7 & 0.0517 & 3 & 30 \\
\hline 8 & 0.9946 & 3 & 3 \\
\hline 9 & 0.9943 & 4 & 6 \\
\hline 10 & -0.0424 & 8 & 18 \\
\hline 11 & 0.9138 & 2 & 4 \\
\hline 12 & 0.9710 & 2 & 2 \\
\hline 13 & 0.9956 & 1 & 2 \\
\hline 14 & 0.9669 & 3 & 4 \\
\hline 15 & 0.7823 & 2 & 43 \\
\hline 16 & 0.9910 & 1 & 2 \\
\hline 17 & 0.9919 & 2 & 2 \\
\hline 18 & -0.9973 & 2 & 45 \\
\hline 19 & 0.9918 & 3 & 3 \\
\hline 20 & -0.0477 & 5 & 86 \\
\hline 21 & 0.9926 & 2 & 4 \\
\hline 22 & 0.9096 & 5 & 4 \\
\hline 23 & 0.9647 & 2 & 4 \\
\hline 24 & -0.7217 & 4 & 53 \\
\hline 25 & 0.9080 & 2 & 5 \\
\hline 26 & 0.9827 & 1 & 2 \\
\hline 27 & 0.8350 & 2 & 6 \\
\hline 28 & -0.8659 & 2 & 28 \\
\hline 29 & 0.9892 & 5 & 4 \\
\hline
\end{tabular}

Tab 1 - Results table with all images

This also shows in the results table, with some images having a large RMSE between operator mean and algorithm. It should be noted, that for the images with a large algorithm RMSE, the algorithm completely missed the points selected by the operators; which is the case in the images where the algorithm fails completely, tracking random pixels, see Fig 8 and Fig 9. These images are noisy with intensities in the same range as the mitral leaflet movement line. These images causes the average RMSE for the algorithm to be relatively high, while the median RMSE is only 4 pixels - giving a representation of how well the algorithm performs with respect to the images of higher quality, where the algorithm performs better, see Fig 10.

For the images where the method works well it presents a nicely tracked line that could be used as a guideline for the clinician when marking the timing points for valve opening and closing. The method could also be extended to place these points automatically, using the colors from the original ultra sound images and the method described by $[1,2]$.
A great advantage of the algorithm is that it tracks a pixel for every column in the image, unlike an operator who will be prone to selecting only the points necessary to roughly describe the mitral valve movement line, not giving a detailed description of the movement line.

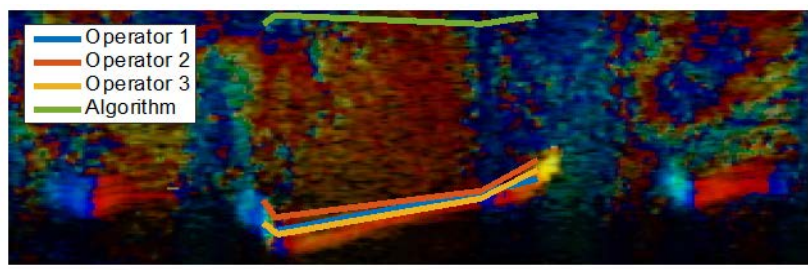

Fig 8 - Bad agreement between operators and algorithm (Image No 20)

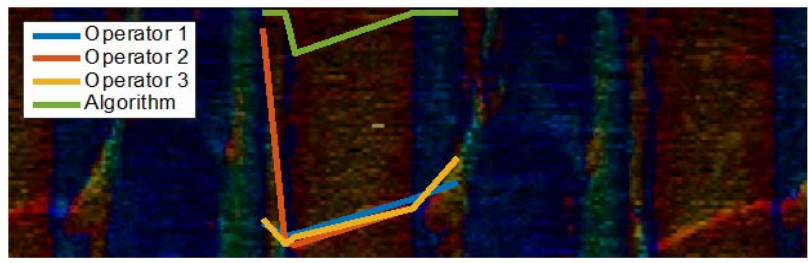

Fig 9 - Bad agreement inter operators and algorithm (Image No 3)

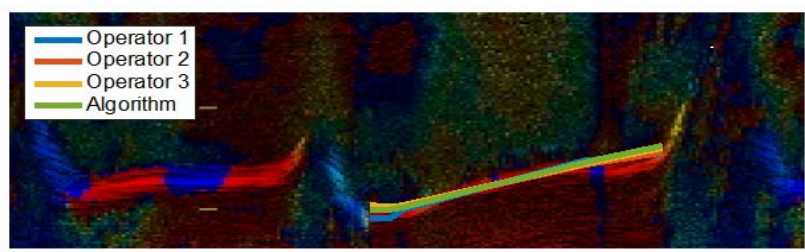

Fig 10 - Good agreement between operators and algorithm (Image No 5). All lines are on top of each other

\section{References}

[1] Kjaergaard J, Hassager C, Oh JK, et al. Measurement of cardiac time intervals by Doppler tissue M-Mode imaging of the anterior mitral leaflet. J Am Soc Echocardiogr 2005; 18: 1058-1065.

[2] Voigt JU, Lindenmeier G, Exner B, et al. Incidence and characteristics of segmental postsystolic longitudinal shortening in normal, acutely ischemic, and scarred myocardium. J Am Soc Echocardiogr 2003; 16: 415-423.

[3] Gonzalez RC, Woods RE. Digital Image Processing (3rd Edition). 2008.

Address for correspondence.

Kasper Sørensen

Aalborg University

Department of Health Science and Technology

Fredrik Bajers Vej 7

ks@hst.aau.dk 